\title{
Myofiber Permeability and Force Production of Rat Muscles Following Eccentric Contractions: The Repeated Bout Effect Depends on the Interval
}

\author{
Keishi Hayao $^{1,2}\left({ }^{\circ}\right.$, Hiroyuki Tamaki $^{3}$ (), Keigo Tamakoshi ${ }^{1}$, Hideaki Takahashi ${ }^{1}$, Hideaki Onishi $^{1}$ \\ ${ }^{1}$ Institute for Human Movement and Medical Sciences, Niigata University of Health and Welfare, Niigata, Japan; \\ ${ }^{2}$ Southern Tohoku General Hospital, Fukushima, Japan; ${ }^{3}$ National Institute of Fitness and Sports in Kanoya, \\ Kagoshima, Japan
}

Correspondence to: Keishi Hayao, hwd18009@nuhw.ac.jp

Keywords: Eccentric Contraction, Muscle Injury, Repeated Bout Effect, Myofiber Membrane Permeability, Maximal Isometric Contraction Tension

Received: November 11, $2020 \quad$ Accepted: December 28, $2020 \quad$ Published: December 31, 2020

Copyright $\odot 2020$ by author(s) and Scientific Research Publishing Inc.

This work is licensed under the Creative Commons Attribution International License (CC BY 4.0).

http://creativecommons.org/licenses/by/4.0/

\section{(c) (i) Open Access}

\section{ABSTRACT}

Past eccentric contraction (ECC)-induced muscle injury reduces the severity of symptoms of subsequent muscle injury; this phenomenon is known as a repeated bout effect (RBE). It has been reported that increases in the duration of the interval between the first and second bouts are linked to weakening of the RBE. However, the histology following the attenuation of the RBE remains unclear. We examined the sustained effects of the second bout with regard to myofiber permeability and muscle force. Sixty-four male rats were randomly assigned to eight groups that varied in the number of exercise sessions and the duration of the interval between the first and second bouts: the non-ECC (Control); the single-injury (Post 1st bout); groups that were allowed to recover for 1, 2, and 4 weeks after a single injury (Pre 2nd bout_1w, Pre 2nd bout_2w, and Pre 2nd bout_4w); and groups that were subjected to second injuries 1, 2, and 4 weeks after the first (Post 2nd bout_1w, Post 2nd bout_2w, and Post 2nd bout_4w). The tibialis anterior was electrically stimulated in each ECC group. Twenty-four hours before muscle sampling, Evans blue dye (EBD) (a marker of myofiber damage) was administered. The maximal isometric contraction tension was measured immediately before sampling. The number of EBD-positive (+) fibers was determined via histological analysis. An RBE was revealed by functional examination at the 1- and 2-week and histological examination at the 1-, 2-, and 4-week time points $(P<0.05)$. In terms of myofiber permeability, prolongation of the interval before the second bout weakened this effect $(P<0.05)$. Experiments with 1-, 2-, and 4-week intervals indicated that prolongation of the 


\section{interval before the second bout weakened the RBE with regard to myofiber permeability.}

\section{INTRODUCTION}

Compared with concentric contraction and isometric contraction, eccentric contraction (ECC) results in greater muscle strength $[1,2]$. On the other hand, ECC more easily causes muscle damage, swelling, and loss of function than these other types of contraction [3-6].

Excessive ECC increases membrane permeability, causes creatine kinase to leak out of myofibers [7], into the plasma, and causes extracellular proteins such as albumin to enter myofibers [8]. Infiltration of extracellular fluid causes myofibers to become swollen and opaque [9]. The main pathways for extracellular fluid entry into myofibers following ECC have been reported to be membrane tears and stretch-activated channels [10]. Evans blue dye (EBD; molar mass 960.8), as a marker of increased myofiber membrane permeability, is a tool for studying ECC-induced muscle damage [8]. EBD is water soluble and membrane impermeable, has high binding affinity for serum albumin, and can be used to detect damaged myofibers. Positive staining for glyoxal-bis (2-hydroxyanil) (GBHA) (a free calcium indicator) has been observed in EBD-stained infiltrated myofibers after ECC [9]. Excessive increases in intracellular calcium ions promote the production of calcium dependent proteases, such as calpain and phospholipases, which causes myofiber membrane damage and disruption in intermediate filament proteins, such as desmin, that link myofibrils laterally to each other [11-14].

Adaptation of damaged skeletal muscle to injury reduces the severity of symptoms of subsequent muscle damage; this phenomenon is known as a repeated bout effect (RBE). It has been reported that the ECC RBE results in a shorter period of muscle strength recovery post ECC, a lower concentration of blood creatine kinase, and less muscle soreness in the second bout than in the initial bout [15-19]. Animal studies investigating ECC have also suggested functional implications (e.g., smaller isometric strength deficits in the second bout than in the first bout) and structural implications (e.g., smaller reductions in muscle cell membrane structural proteins, such as dystrophin, and less disruption of inter-mediate filament proteins, such as desmin) [20,21]. In addition, fewer myofibers with increased membrane permeability are observed in the second bout than in the first bout, and a history of ECC-induced exercise injury attenuates calpain activation [22].

A previous study reported that prolonging the duration of the interval between the first and second bouts with the same load strength weakened the RBE (e.g., it weakened the attenuation of changes in peak plasma creatinine kinase activity and the recovery of maximal isometric force post exercise) [19]. However, in that human study, the histological changes following the attenuation of the RBE were unclear. Animal studies conducted with 2- or 4-week intervals have used different ECC intervention intensities and examined different target muscles [21,23], and the associated persistence and attenuation have remained unclear. Thus, the ECC RBE-associated histological changes that occur in skeletal muscle when the interval is prolonged need to be elucidated. Moreover, few investigations have assessed the recovery process after the first bout, increases in myofiber permeability, the recovery of myofibers, and the muscle function of the same skeletal muscle immediately prior to the second bout. In the rat tibialis anterior (TA) muscle, the cross-sectional areas of myofibers have been found to decrease after ECC. This myofiber size decrease is caused by preferential ECC-related damage to type IIb myofibers with large cross-sectional areas rather than type I or IIa myofibers [24]. In addition, calcium uptake during contractions is higher in fast-twitch myofibers than in slow-twitch myofibers [25], and fast-twitch fibers are more susceptible to ECC-induced muscle injury than slow-twitch fibers [26]. Myofiber regeneration can be evaluated using developmental myosin heavy chain (d-MHC) as an indicator, and d-MHC reactivity has been used to assess myofibers after ECC-induced muscle injury [27, 28], and in denervated muscles [29, 30].

In this study, we observed histological changes (e.g., injury, regeneration) and muscle function changes that occur during the post-first bout period immediately prior to the second bout (pre-second bout) with 1-, 2-, and 4-week intervals between the two bouts and examined the sustained effects of the second bout relative to the histological changes and muscle function. 


\section{METHODS}

\subsection{Animals}

Sixty-four male Fischer 344 rats (CLEA Japan, Inc., Tokyo, Japan) were housed in standard cages in a temperature-controlled room under the following conditions: a temperature of $23^{\circ} \mathrm{C} \pm 2{ }^{\circ} \mathrm{C}$, a humidity of $55 \% \pm 5 \%$, and a 12 -h light-dark cycle. The rats had ad libitum access to CE-2 rodent chow (CLEA Japan) and water. All procedures were performed in accordance with the Guiding Principles for the Care and Use of Animals in the Field of Physiological Sciences published by the Physiological Society of Japan. This study was approved by the Animal Study Committee of the National Institute of Fitness and Sports and the Animal Study Committee of Niigata University of Health and Welfare. This study was carried out at the Laboratory of Niigata University of Health and Welfare from 2016 to 2018.

\subsection{Experimental Protocol}

At the age of 12 weeks, rats weighing $251.58 \pm 16.79 \mathrm{~g}$ were randomly assigned to one of eight groups, which differed in the number of exercise sessions (bouts) and the duration of the interval between the first and second bouts. These groups included the non-ECC (Control) group $(n=8)$; the single-injury (Post 1 st bout) group $(n=8)$; groups allowed to recover for 1,2 , and 4 weeks after a single injury (the Pre 2nd bout_1w, Pre 2nd bout_2w, and Pre 2nd bout_4w groups; $n=8$ per group); and groups subjected to second injuries at 1-, 2-, and 4-week intervals (the Post 2nd bout_1w, Post 2nd bout_2w, and Post 2nd bout_4w groups; $n=8$ per group) (Figure 1 ). The appropriate sample size ( $n=8$ per group) was calculated with reference to EBD-positive fiber data from a previous study conducted in our laboratory using the following formula [9]:

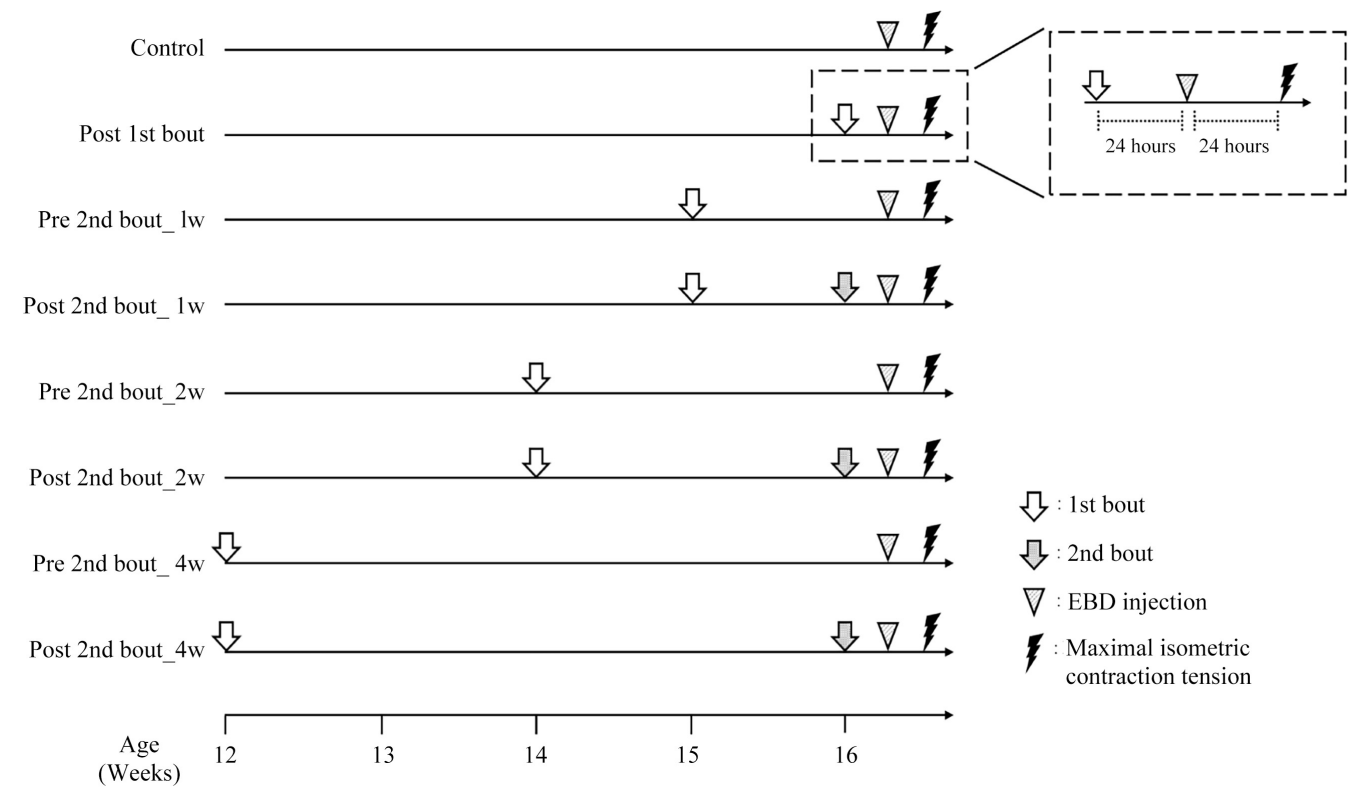

Figure 1. Experimental protocol. Groups: Control (non-ECC; $n=8$ ); Post 1st bout (single injury; $n=8$ ); Pre 2nd bout_1w, Pre 2nd bout_2w, and Pre 2nd bout_4w (recovery for 1, 2, and 4 weeks after a single injury; $n=8$ per group); and Post 2nd bout_1w, Post 2nd bout_2w, and Post 2nd bout_4w (2nd injuries at 1-, 2-, and 4-week intervals; $n=8$ per group). At the age of 16 weeks, all rats were anaesthetized, and muscle samples were obtained. EBD was intraperitoneally administered $24 \mathrm{~h}$ prior to the collection of muscle samples, and the maximal isometric contraction tension was measured immediately prior to muscle sampling. The first and second bouts of eccentric contraction were set to the same load strength. ECC, eccentric contraction; EBD, Evans blue dye. 


$$
n=(r+1) / r \times \sigma^{2}\left(Z_{\alpha / 2}+Z_{\beta}\right)^{2} / \Delta^{2},
$$

where $r$ is the ratio of the larger group size to the smaller group size, $\sigma$ is the standard deviation, $\Delta$ is the effect size, $\alpha=0.05$ (for $95 \%$ confidence), $\beta=0.2$ (for $80 \%$ power), $Z_{\alpha / 2}=1.96$, and $Z_{\beta}=0.84$.

At the age of 16 weeks, all rats were anaesthetized, and muscle samples were obtained. EBD was intraperitoneally administered twenty-four hours prior to the collection of muscle samples, and the maximal isometric contraction tension was measured immediately prior to muscle sampling. The first and second bouts of ECC were set to the same load strength and were performed as described in a previous study [9]. Briefly, the left TA muscles of the rats in each ECC group were electrically stimulated under $2 \%$ isoflurane inhalation anaesthesia. The rats were placed supine on the platform of a custom-made apparatus. The left foot was attached to a footplate connected to a servomotor (Tower Pro SG-90; Umemoto, Tokyo, Japan), and the ankle joint was set at $0^{\circ}$ dorsiflexion. A pair of surface electrodes were attached to the shaved skin over the TA muscle. Percutaneous stimulation was performed using an electrostimulator (SEN-7203; Nihon Kohden, Tokyo, Japan) and isolator (SS-201J; Nihon Kohden). The electrical stimulation parameters were as follows: an intensity of $30 \mathrm{~V}$, a frequency of $100 \mathrm{~Hz}$ and a pulse width of $500 \mu$ s to induce submaximal tetanic contraction for $2 \mathrm{~s}$. The footplate was moved in synchrony with the electrical stimulator and the time-delay device (Raspberry Pi 2 Model B; Raspberry Pi Foundation, Cambridge, UK). ECC were induced at an angular velocity of $200 \%$ s. One ECC set was composed of 10 contractions every 10 $\mathrm{s}$, and eight sets were acquired per animal at 3-min intervals.

\subsection{Injection of EBD}

To assess the myofibers responsible for increasing membrane permeability, rats received an intraperitoneal injection of 1\% EBD solution (E2129; Sigma-Aldrich, St. Louis, MO, USA) at a volume of $1 \%$ of the body mass ( $1 \mathrm{mg}$ EBD/0.1 mL phosphate-buffered saline [PBS]/10g of body mass) $24 \mathrm{~h}$ prior to TA muscle collection $[9,31]$.

\subsection{Measurement of Maximal Isometric Contraction Tension}

TA muscle maximal isometric contraction tension was measured at 16 weeks of age $24 \mathrm{~h}$ after injection of EBD (Control, $n=6$; Post 1st bout, $n=7$; Pre 2nd bout_1w, $n=8$; Post 2nd bout_1w, $n=7$; Pre 2nd bout_2w, $n=6$; Post 2nd bout_2w, $n=4$; Pre 2nd bout_4w, $n=4$; and Post 2nd bout_4w, $n=4$ ). Maximal isometric contraction tension was measured in the Post 1st bout and Post 2nd bout groups 2 days after the last ECC intervention. Maximal isometric contraction tension was measured separately in the Pre 2nd bout groups (Pre 2nd bout_1w, Pre 2nd bout_2w, and Pre 2nd bout_4w) each week after the first ECC intervention. Measurement was performed as previously described [32, 33]. Briefly, under isoflurane anaesthesia, rats were placed on a working platform with restraining bars and pins at the knee and ankle joints. The TA muscle distal tendon was oriented along the natural pull of the muscle and attached to an isometric transducer (TB-654T; Nihon Kohden) that was secured using 4-0 suture silk on a three-dimensional (3D) drive precision stage. Maximal isometric contraction tension was determined from the average of three tetanic contractions. The signals were sampled via a PowerLab A/D converter (AD Instruments, Nagoya, Japan) with a sampling frequency of $2 \mathrm{kHz}$.

\subsection{Muscle Sampling}

At the end of the experiment, following the measurement of tension, the rats were anaesthetized with sodium pentobarbital ( $50 \mathrm{mg} / \mathrm{kg}$ body weight), and their TA muscles were collected and weighed. For histological analyses, the TA muscles were mounted with optimal cutting temperature compound, frozen in isopentane cooled using liquid nitrogen and stored at $-80^{\circ} \mathrm{C}$ until use.

\subsection{Histological Analysis}

Frozen transverse sections $(10 \mu \mathrm{m})$ were cut from the middle portion of the TA muscle at $-20^{\circ} \mathrm{C}$ us- 
ing a cryostat (CM3050S; Leica, Wetzlar, Germany). The sections were mounted on silanized slides, and signal detection analysis of EBD (a marker of myofibers with membrane damage caused by ECC) was performed. Images of transverse sections of the muscle belly were acquired using fluorescence microscopy (BZ-X710; KEYENCE, Osaka, Japan). The myofibers in the muscle belly were counted using Image-Pro Premier software (Media Cybernetics, Rockville, MD, USA) to quantify the number of EBD-positive (+) fibers. The transverse sections stained with hematoxylin and eosin (H\&E) for histological observation. For immunohistochemical analysis of TA muscle tissues, sections were fixed in ice-cold $4 \%$ paraformaldehyde for $15 \mathrm{~min}$. The sections were blocked with $10 \%$ normal goat serum (NGS) and 1\% Triton X-100 in PBS at room temperature for $1 \mathrm{~h}$ and then washed twice (5 min each) in PBS. Subsequently, the sections were incubated for $16-20 \mathrm{~h}$ at $4^{\circ} \mathrm{C}$ with primary antibodies against dystrophin (ab15277; 1:500 dilution; Abcam, Tokyo, Japan) and d-MHC (MHCD; 1:20 dilution; Novocastra, Newcastle upon Tyne, UK) (a marker of myofiber regeneration) in PBS containing 2.5\% NGS and $0.1 \%$ Triton X-100. The sections were washed several times with PBS, incubated with an Alexa Fluor 488 (ab150117; 1:500 dilution; Abcam) or 568 (ab175471; 1:500 dilution; Abcam)-conjugated secondary antibody diluted with PBS containing 2.5\% NGS and $1 \%$ Triton $\mathrm{X}-100$ for $1 \mathrm{~h}$ at room temperature, and finally mounted using VECTASHIELD mounting medium with 4',6-diamidino-2-phenylindole (H-1200; Vector Laboratories, Burlingame, CA, USA). Immunofluorescence of the transverse sections was detected using a light/fluorescence microscope (BZ-X710; KEYENCE). The numbers of d-MHC-labelled (+) and dystrophin+ myofibers in five $500 \times 700 \mu \mathrm{m}$ fields of deep, superficial, middle, inside-middle, and outside-middle layers in eight transverse sections of TA muscle were determined manually.

\subsection{Statistical Analysis}

All data are presented as the mean \pm standard deviation. Data sets were analysed using one-way analysis of variance (ANOVA) followed by either a post hoc Tukey-Kramer test or a Kruskal-Wallis test followed by a Steel-Dwass test or a Steel test (BellCurve for Excel, Social Survey Research Information Co., Ltd., Tokyo, Japan), depending on the normality of the data distribution. P-values $<0.05$ denoted statistical significance.

\section{RESULTS}

\subsection{Muscle Wet Weight}

TA muscle weight relative to body weight was significantly higher in the Post 1st bout, Pre 2nd bout_4w, and Post 2nd bout_4w groups than in the Control group $(\mathrm{P}<0.05)$ (Control $=1.67 \pm 0.05$, Post 1 st bout $=2.05 \pm 0.08$, Pre 2 nd bout_4 $\mathrm{w}=2.02 \pm 0.13$, and Post 2 nd bout_4w $=2.15 \pm 0.08)$. In contrast, TA muscle weight relative to body weight was lower in the Pre 2nd bout_1w and Post 2nd bout_1w groups than in the Control group $(\mathrm{P}<0.05)$ (Pre 2nd bout_1w $=1.31 \pm 0.21$, and Post 2nd bout_1w $=1.24 \pm$ $0.21)$. There were no significant differences in the Pre 2 nd bout_2w and Post 2nd bout_2w groups compared with the Control group (Pre 2nd bout_2w $=1.63 \pm 0.09$ and Post 2nd bout_2w $=1.71 \pm 0.13$ ) (Figure 2).

\subsection{Maximal Isometric Contraction Tension}

TA maximal isometric contraction tension relative to muscle wet weight was significantly lower in the Post 1st bout group than in the Control group $(\mathrm{P}<0.05)$. There were no significant differences among the Pre 2nd bout_1w, Pre 2nd bout_2w, Pre 2nd bout_4w, and Control groups. The tension was significantly higher in the Post 2nd bout_1w and Post 2nd bout_2w groups than in the Post 1st bout group $(\mathrm{P}<0.05)$. There were no significant differences among the Post 2nd bout_1w, Post 2nd bout_2w, and Post 2nd bout_4w groups (Control $=0.016 \pm 0.003$, Post 1st bout $=0.004 \pm 0.001$, Pre 2nd bout_1w $=0.013 \pm 0.002$, Post 2nd bout_1w $=0.011 \pm 0.003$, Pre 2nd bout_2w $=0.015 \pm 0.001$, Post 2nd bout_2w $=0.011 \pm 0.002$, Pre 2nd bout_4w $=0.014 \pm 0.001$, and Post 2nd bout_4w $=0.007 \pm 0.002$ ) (Figure 3 ). The decreases in 
maximal isometric contraction tension according to the bout interval in the different groups were as follows: Post 2nd bout_1w/Pre 2nd bout_1 $\mathrm{w}^{\star} 100=15 \%$, Post 2 nd bout_2w/Pre 2 nd bout_2 $\mathrm{w}^{*} 100=31 \%$, and Post 2nd bout_4w/Pre 2nd bout_4 $4 \mathrm{w}^{\star} 100=47 \%$.

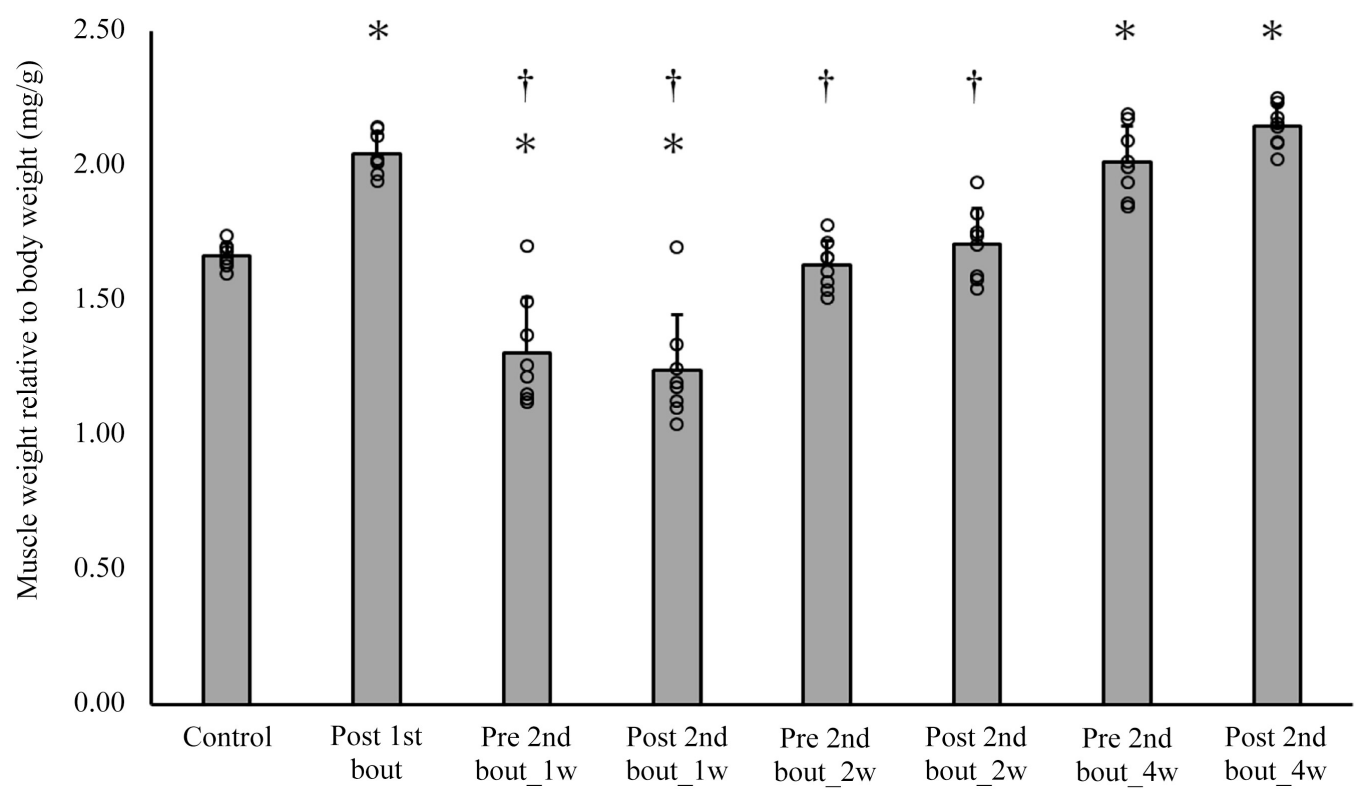

Figure 2. Muscle wet weight of the tibialis anterior muscle relative to body weight in each group. ${ }^{*} \mathrm{P}<0.05$ versus Control; $\uparrow \mathrm{P}<0.05$ versus $P$ ost 1 st bout. The values are presented as the mean $\pm \mathrm{SD}$. SD, standard deviation.

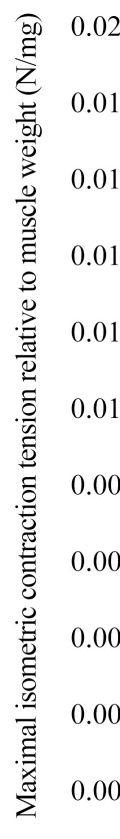

.020
.018
.016
.014
0.012
0.008
0.006
0.002
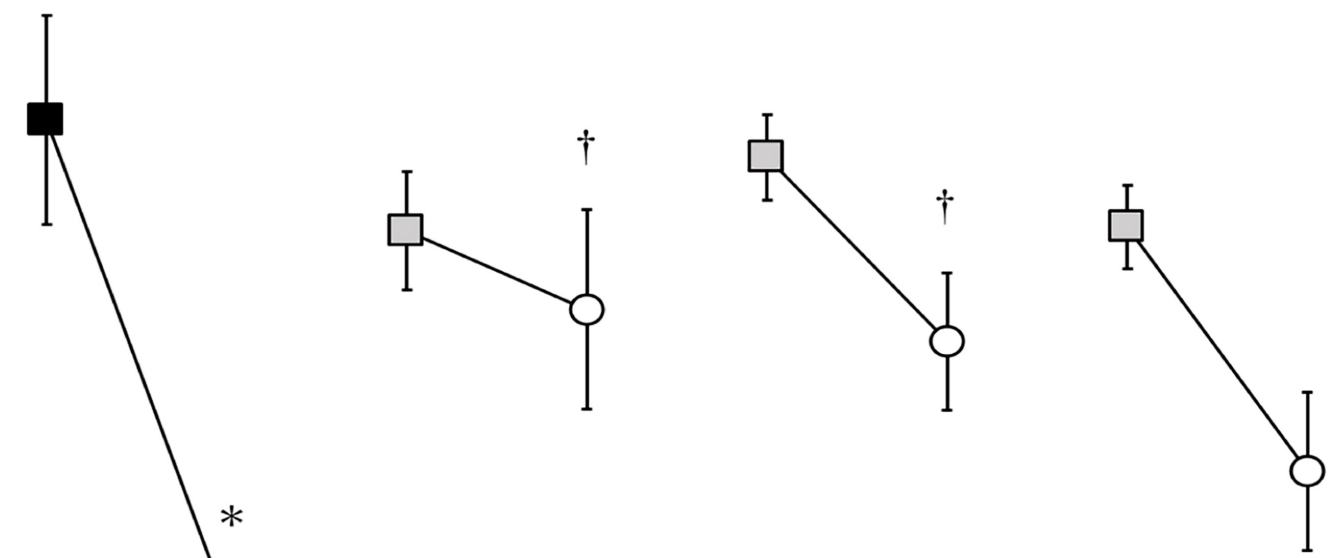

Figure 3. Maximal isometric contraction tension relative to muscle wet weight in each group. ${ }^{*} \mathbf{P}$ $<0.05$ versus Control; $\uparrow P<0.05$ versus Post 1 st bout. The values are presented as the mean \pm SD. $\mathrm{SD}$, standard deviation. 


\subsection{Number of EBD+ Fibers and Histological Analysis}

EBD+ fibers were observed in the Post 1st bout, Post 2nd bout_1w, Post 2nd bout_2w, and Post 2nd bout_4w groups. In the Control group, almost no EBD+ fibers were detected. Similarly, EBD+ fibers were rarely detected in the Pre 2nd bout_1w, Pre 2nd bout_2w, and Pre 2nd bout_4w groups. Among the second-bout groups, there were fewer EBD+ fibers in the Post 2nd bout_1w, Post 2nd bout_2w, and Post 2nd bout_4w groups than in the Post 1 st bout group $(\mathrm{P}<0.05)$. Moreover, there were significantly more fibers in the Post 2nd bout_4w group than in the Post 2 nd bout_1w and Post 2nd bout_2w groups $(\mathrm{P}<$ $0.05)$ (Control $=0.25 \pm 0.46$ cells, Post 1 st bout $=2214.38 \pm 535.99$ cells, Pre 2 nd bout $\_1 \mathrm{w}=1.25 \pm 1.67$ cells, Post 2 nd bout_1w $=3.00 \pm 2.27$ cells, Pre 2 nd bout_2w $=0.75 \pm 0.89$ cells, Post 2 nd bout_2w $=80.63$ \pm 85.62 cells, Pre 2nd bout_4w $=1.50 \pm 2.27$ cells, and Post 2 nd bout_4w $=839.88 \pm 335.99$ cells) (Figures 4A-I). Using H-E Staining, we examined myofiber damage and recovery in each group. Swollen myofibers, necrotic myofibers and infiltration of inflammatory cells observed in the Post 1st bout, Post 2nd bout_2w, and Post 2nd bout_4w groups (Figure 5).
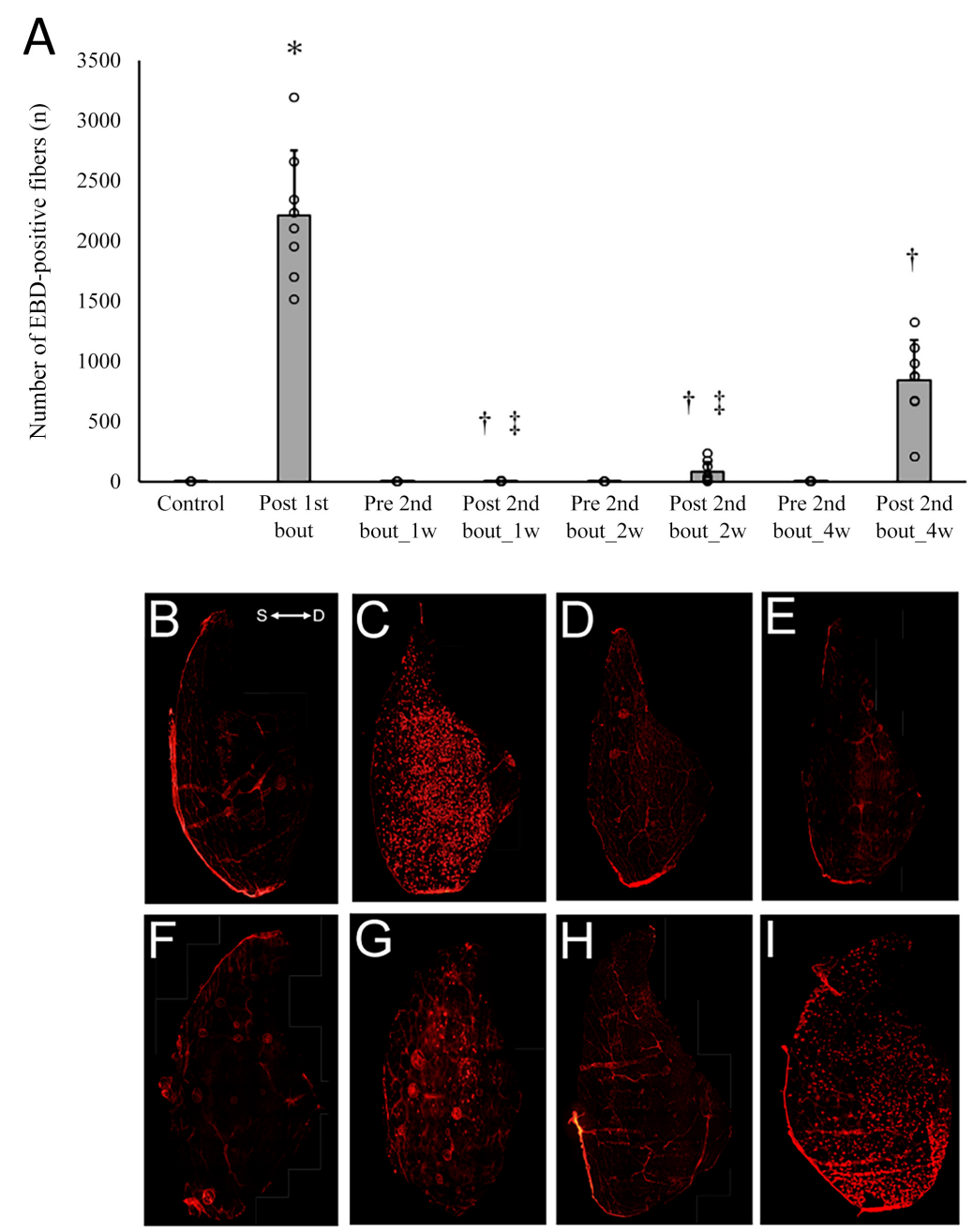

Figure 4. Number of EBD-positive (+) myofibers in each group. (a) Number of EBD+ myofibers in each group. ${ }^{\star} \mathrm{P}<0.05$ versus Control; $\uparrow \mathrm{P}<0.05$ versus Post 1 st bout; $\ddagger \mathrm{P}<0.05$ versus Post 2nd bout_4w. The values are presented as the mean \pm SD. EBD, Evans blue dye; SD, standard deviation. (b-i) Histological images of EBD+ myofibers in each group. (b) Control, (c) Post 1st bout, (d) Pre 2nd bout_1w, (e) Post 2nd bout_1w, (f) Pre 2nd bout_2w, (g) Post 2nd bout_2w, (h) Pre 2nd bout_4w, and (i) Post 2nd bout_4w. S, D; Superficial, deep side of the TA. 


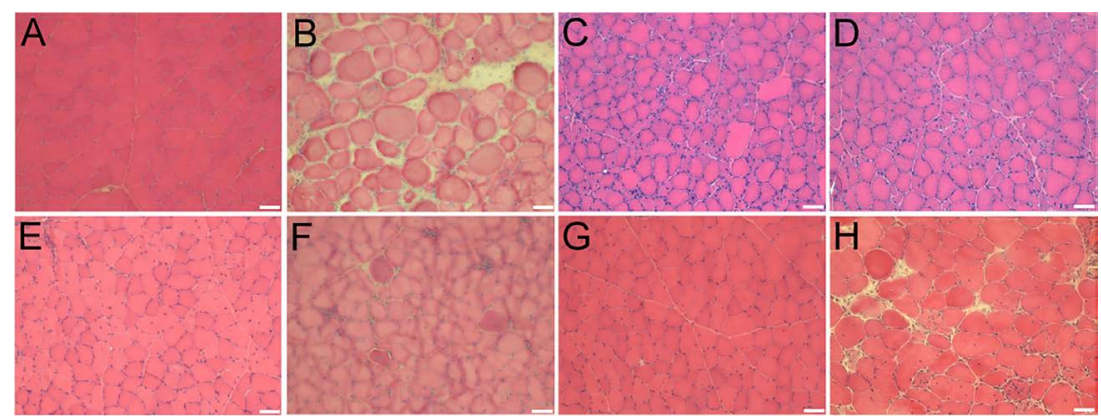

Figure 5. Image of cross-sections of tibialis anterior muscles stained for Hematoxylin and Eosin (H\&E) in each group. (a) Control, (b) Post 1st bout, (c) Pre 2nd bout_1w, (d) Post 2nd bout_1w, (e) Pre 2nd bout_2w, (f) Post 2nd bout_2w, (g) Pre 2nd bout_4w, and (h) Post 2nd bout_4w groups. Scale bar $=50 \mu \mathrm{m}$.

\section{4. d-MHC +/Dystrophin + Fibers}

There were significantly more d-MHC+/dystrophin+ fibers in the Pre 2nd bout_1w and Post 2nd bout_1w groups than in the Post 1st bout group $(\mathrm{P}<0.05)$. No significant difference was found between the Pre 2nd bout_1w and Post 2nd bout_1w groups. In contrast, d-MHC+/dystrophin+ fibers were rarely detected in the Control, Post 1st bout, Pre 2nd bout_2w, Post 2nd bout_2w, Pre 2nd bout_4w, and Post 2nd bout_4w groups (Control $=$ not detected, Post 1st bout $=1.25 \pm 1.75$ cells, Pre 2nd bout_1w $=16.63 \pm$ 9.01 cells, Post 2 nd bout_1w $=11.00 \pm 6.59$ cells, Pre 2nd bout_2w $=0.13 \pm 0.35$ cells, Post 2nd bout_2w $=$ $0.50 \pm 0.76$ cells, Pre 2nd bout_4w $=0.13 \pm 0.35$ cells, and Post 2 nd bout $4 \mathrm{w}=$ not detected) (Figures 6-8) Moreover, myofibers with increased membrane permeability were rarely observed, and dystrophin+ fibers were observed in almost all areas of the TA transverse sections in the Pre 2nd bout_1w, Pre 2nd bout_2w, and Pre 2nd bout_4w groups (Figure 7).

\section{DISCUSSION}

This study investigated the following: 1) the histological changes, muscle swelling and muscle function prior to the second bout of ECC at 1-, 2-, and 4-week intervals; and 2) the effects of repeated bouts of ECC on myofiber damage and force production dependent on the interval between the first and second bouts.

The muscle wet weight was found to increase $48 \mathrm{~h}$ after ECC due to swelling and tissue damage in the rat TA muscles. Extracellular fluid influx into myofibers is induced by mechanical stress due to ECC, appear swollen and opaque $48 \mathrm{~h}$ after ECC, EBD+ fibers (as a marker of increased myofiber membrane permeability) swell approximately twice as much as non-EBD fibers, and muscle wet weight and EBD+ fiber numbers are increased $48 \mathrm{~h}$ after ECC $[9,24,34]$. It has been reported that the several pathways for extracellular fluid entry to myofibers following ECC, the main pathways are cell membrane lesions (membrane tears) and stretch-activated channels (SACs) [10]. EBD exhibits high binding affinity for albumin, and albumin binds avidly to calcium [35]. This complex flows into damaged myofibers following increases in membrane permeability. The concentration of intracellular calcium ions $\left(\left[\mathrm{Ca}^{2+}\right] \mathrm{i}\right)$ increases $48 \mathrm{~h}$ after ECC [34], calcium-dependent protease (calpain) becomes activated with increases in $\left[\mathrm{Ca}^{2+}\right] \mathrm{i}[36]$, and activated calpain breaks down cytoskeleton-related proteins and contraction-related proteins in myofibers [37]. Moreover, negative reaction for dystrophin and desmin on immunostaining has been reported to be observed in EBD infiltrated myofibers $48 \mathrm{~h}$ after ECC [9].

After myofiber damage accompanied by increased membrane permeability is caused by ECC, myofiber regeneration occurs, and d-MHC immunoreactivity is observed [27, 28]. In this study, there were significantly more d-MHC+/dystrophin + myofibers in the Pre 2nd bout_1w and Post 2nd bout_1w groups than in the Post 1st bout group, and membrane-permeable myofibers were rarely observed 1 week after 
ECC. Previous studies have also shown that the number of $\mathrm{d}-\mathrm{MHC}+$ myofibers is significantly elevated $\geq 5$ days after ECC, that there are significant numbers of positive myofibers by day 7 and that there are almost no positive myofibers by day $12[27,28]$. In a previous study have reported muscle tension to recover to its baseline level one week after ECC [31]. In other studies, however, recovery of muscle tension has been reported to be inadequate 3 weeks after ECC [27]. The lengths of the recovery periods for plasma creatine kinase activity and muscle strength after ECC increase as the severity of muscle injury increases [38, 39].

An RBE was observed with regard to increases in myofiber permeability when 1-, 2-, and 4-week intervals between bouts, force production when 1- and 2-week intervals between bouts were used. Prolongation of the interval prior to the second bout weakened the effect on myofiber permeability. Previous human studies have similarly reported that prolonging the interval weakens RBEs in terms of blood creatine kinase activity and maximal isometric force [19]. In rodent studies involving two bouts of ECC separated by 2 - and 4 -week intervals, eccentric torque and isometric tetanic torque have been found to be reduced to a greater extent during the first versus the second ECC bout $[20,21,23]$. However, among these previous rodent studies, the target muscles and intensity of ECC have differed. The findings of our study, which was conducted with the same intensity and the same target muscle for both bouts, revealed that the degree of RBE was associated with the interval duration. Cellular influx of extracellular fluids occurs through cell membrane defects after ECC as well as through stretch-activated channels, transient receptor potential channels [10], and aquaporins (AQPs). In particular, AQP4 is thought to play roles in adjusting osmotic pressure and intracellular volume $[40,41]$ and is also related to the expression of dystrophin. AQP4 has been reported to be absent in dystrophin-deficient Duchenne muscular dystrophy model (mdx) mice [42]. A study involving administration of AQP4 in a muscle injury model reported an immunonegative reaction as early as 2 weeks after muscle injury. Notably, a significant positive response was observed 4 weeks later [43]. While an immunopositive response to dystrophin was observed 1 week after ECC in this study, there was almost no intracellular infiltration of EBD, and only minor infiltration was observed 2 weeks after ECC, although significantly increased infiltration was observed by 4 weeks. Furthermore, in many cases, AQP4 was selectively expressed in fast-twitch myofibers. The rat TA tissues used in this study showed widespread distribution of fast-twitch myofibers. Therefore, we suggest that the expression of AQP4 after the regeneration of myofibers is involved in the RBE due to its effects on membrane permeability.

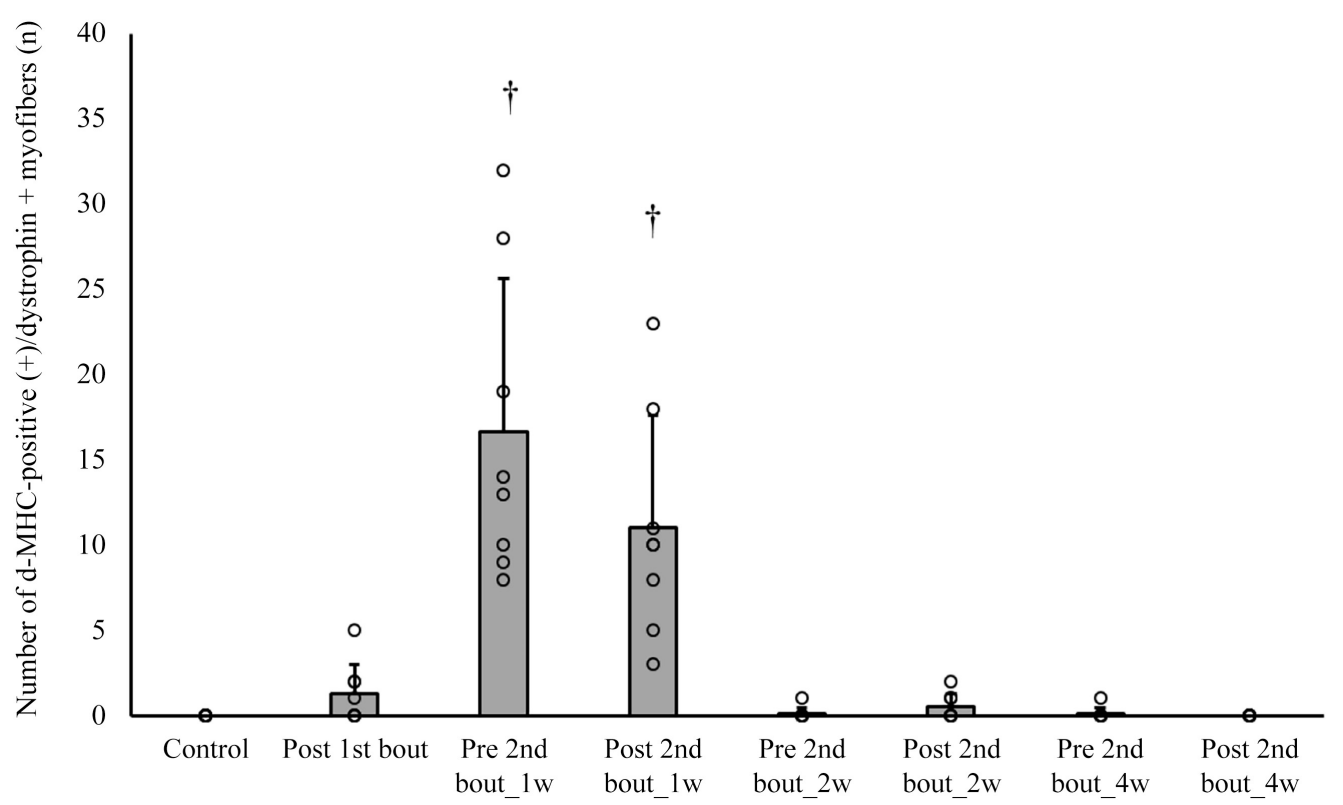

Figure 6. Number of $\mathrm{d}-\mathrm{MHC}+$ and dystrophin + myofibers in each group. $\uparrow \mathrm{P}<$ 0.05 versus Post 1 st bout. The values are presented as the mean \pm SD. d-MHC, developmental myosin heavy chain; SD, standard deviation. 


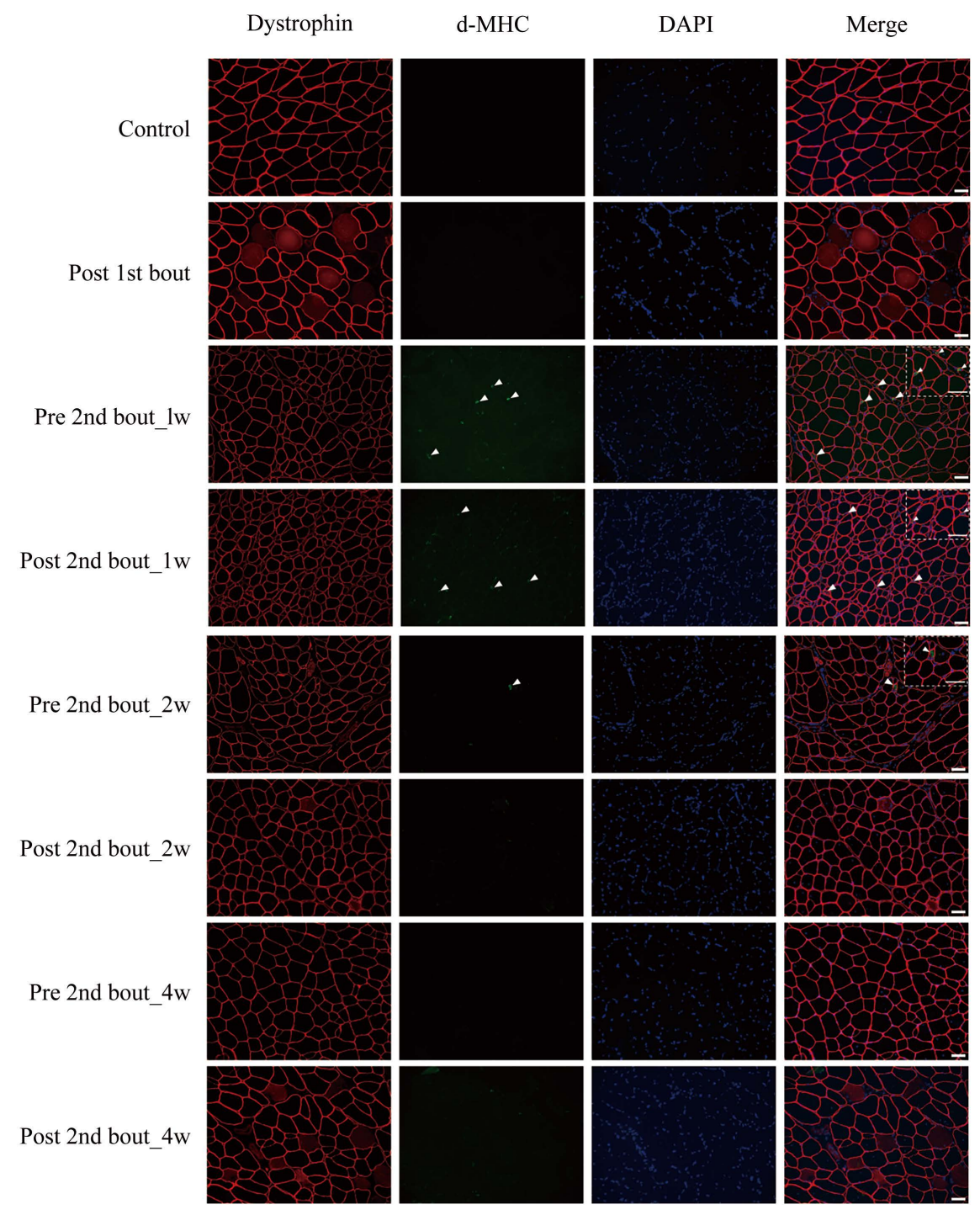

Figure 7. Image of immunostained tibialis anterior muscles cross-sections from each group. Red indicates dystrophin+ areas. Green indicates developmental myosin heavy chain (d-MHC)+ fibers. Blue indicates 4',6-diamidino-2-phenylindole (DAPI). The arrows indicate d-MHC+ and dystrophin+ myofibers. The inset images are high-magnification images of the d-MHC+ and dystrophin + myofibers. Scale bar $=50 \mu \mathrm{m}$.

In this study, myofibers with increased membrane permeability were rarely observed, whereas dystrophin-immunoreactive fibers were observed in almost all areas of the TA transverse sections in the Pre 2nd bout_1w, Pre 2nd bout_2w, and Pre 2nd bout_4w groups. The numbers of d-MHC+/dystrophin+ myofibers were significantly elevated in the Pre 2nd bout_1w and Post 2nd bout_1w groups. It has been reported that regenerated myofibers are present in denervated muscles $[29,30]$ and that denervation reversibly affects the domains of the membrane systems involved in excitation-contraction coupling [44]. Fast-velocity ECC $\left(180^{\circ} / \mathrm{s}\right)$ induces functional and structural damage to nerves [45]. In our study, nerve injury appeared 7 days after ECC, suggesting that d-MHC was expressed under conditions of injury. In addition, there was no significant difference in the number of d-MHC+ myofibers between the Pre 2nd bout_1w and Post 2nd bout_1w groups, suggesting that d-MHC+ fibers are resistant to damage in the early stages after ECC. 

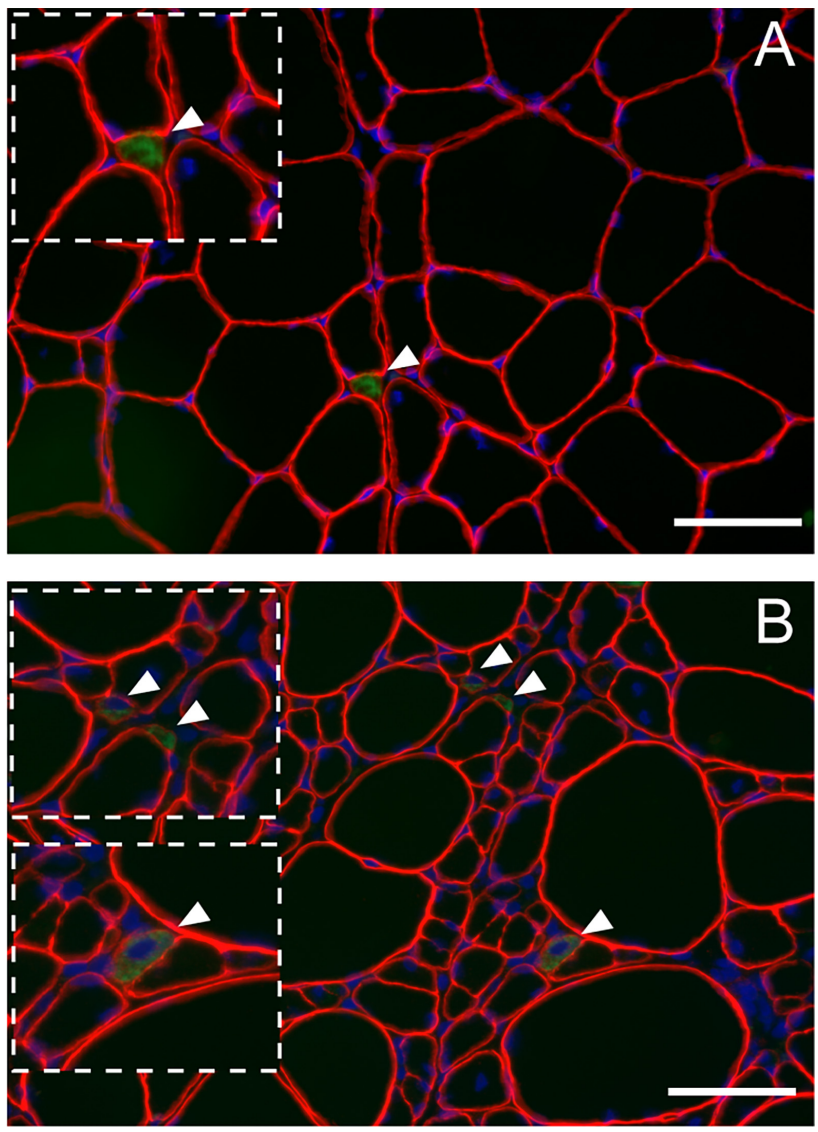

Figure 8. Higher-magnification images of immunostained tibialis anterior muscles cross-sections of d-MHC expressed in the cytoplasm of myofibers (inside dystrophin-positive plasma membrane). Red indicates dystrophin+ areas. Green indicates d-MHC+ fibers. Blue indicates 4',6-diamidino-2-phenylindole (DAPI). The arrows indicate d-MHC+ and dystrophin+ myofibers. (a) merged images in the Pre 2nd bout_1w group and (b) merged images in the Post 2nd bout_1w group. Scale bar $=50 \mu \mathrm{m}$.

A limitation of this study is that we did not examine cellular influx of extracellular fluids through stretch-activated channels, transient receptor potential channels or AQPs. In addition, previous reviews have suggested that the potential mechanism of RBE involves neural adaptations, connective tissues, and myofiber structural remodeling of the extracellular matrix [46, 47]. However, the details of the mechanism are unknown and should be investigated. Moreover, we did not determine the molecular parameters in the present study. This study observed an RBE in terms of myofiber permeability. These results provide, for the first time, evidence that prolongation of the interval between the first and second bouts of ECC weakens the RBE with regard to increased myofiber permeability.

\section{CONCLUSION}

This study observed an RBE in terms of muscle function and histology. Experiments with 1-, 2-, and 4 -week intervals revealed that prolongation of the interval between the first and second bouts of ECC weakened the RBE with regard to increased myofiber permeability. For the first time, this study provides information on basic changes that occur with prolongation of the interval between the first and second bouts: namely, weakening of the RBE associated with increases in myofiber permeability. The findings may aid in the development of strategies for prevention of muscle injury during physical rehabilitation, 
sports and fitness pursuits for health promotion. Further study is needed for a more complete understanding of the mechanism for the RBE on muscle damage. Especially molecular-level analysis would reveal valuable new information on the molecular mechanisms underlying RBE on muscle damage.

\section{CONFLICTS OF INTEREST}

The authors declare no conflicts of interest regarding the publication of this paper.

\section{REFERENCES}

1. Adams, G.R., Cheng, D.C., Haddad, F. and Baldwin, K.M. (2004) Skeletal Muscle Hypertrophy in Response to Isometric, Lengthening, and Shortening Training Bouts of Equivalent Duration. Journal of Applied Physiology, 96, 1613-1618. https://doi.org/10.1152/japplphysiol.01162.2003

2. Ashida, Y., Himori, K., Tatebayashi, D., Yamada, R., Ogasawara, R. and Yamada, T. (2018) Effects of Contraction Mode and Stimulation Frequency on Electrical Stimulation-Induced Skeletal Muscle Hypertrophy. Journal of Applied Physiology, 124, 341-348. https://doi.org/10.1152/japplphysiol.00708.2017

3. Beaton, L.J., Tarnopolsky, M.A. and Phillips, S.M. (2002) Contraction-Induced Muscle Damage in Humans Following Calcium Channel Blocker Administration. The Journal of Physiology, 544, 849-859. https://doi.org/10.1113/jphysiol.2002.022350

4. Crameri, R.M., Aagaard, P., Qvortrup, K., Langberg, H., Olesen, J. and Kjaer, M. (2007) Myofibre Damage in Human Skeletal Muscle: Effects of Electrical Stimulation Versus Voluntary Contraction. The Journal of Physiology, 583, 365-380. https://doi.org/10.1113/jphysiol.2007.128827

5. Newham, D.J., McPhail, G., Mills, K.R. and Edwards, R.H. (1983) Ultrastructural Changes after Concentric and Eccentric Contractions of Human Muscle. Journal of the Neurological Sciences, 61, 109-122. https://doi.org/10.1016/0022-510X(83)90058-8

6. Nosaka, K., Newton, M. and Sacco, P. (2002) Responses of Human Elbow Flexor Muscles to Electrically Stimulated Forced Lengthening Exercise. Acta Physiologica Scandinavica, 174, 37-145. https://doi.org/10.1046/j.1365-201X.2002.00936.x

7. Nosaka, K., Sakamoto, K., Newton, M. and Sacco, P. (2001) The Repeated Bout Effect of Reduced-Load Eccentric Exercise on Elbow Flexor Muscle Damage. European Journal of Applied Physiology, 85, 34-40. https://doi.org/10.1007/s004210100430

8. Hamer, P.W., McGeachie, J.M., Davies, M.J. and Grounds, M.D. (2002) Evans Blue Dye as an in Vivo Marker of Myofibre Damage: Optimising Parameters for Detecting Initial Myofibre Membrane Permeability. Journal of Anatomy, 200, 69-79. https://doi.org/10.1046/j.0021-8782.2001.00008.x

9. Hayao, K., Tamaki, H., Nakagawa, K., Tamakoshi, K., Takahashi, H., Yotani, K., Ogita, F., Yamamoto, N. and Onishi, H. (2018) Effects of Streptomycin Administration on Increases in Skeletal Muscle Fiber Permeability and Size Following Eccentric Muscle Contractions. The Anatomical Record, 301, 1096-1102.

https://doi.org/10.1002/ar.23770

10. Allen, D.G., Whitehead, N.P. and Yeung, E.W. (2005) Mechanisms of Stretch-Induced Muscle Damage in Normal and Dystrophic Muscle: Role of Ionic Changes. The Journal of Physiology, 567, 723-735. https://doi.org/10.1002/ar.23770

11. Sonobe, T., Inagaki, T., Poole, D.C. and Kano, Y. (2008) Intracellular Calcium Accumulation Following Eccentric Contractions in Rat Skeletal Muscle in Vivo: Role of Stretch-Activated Channels. American Journal of Physiology Regulatory, Integrative and Comparative Physiology, 294, R1329-R1337. https://doi.org/10.1152/ajpregu.00815.2007

12. Yeung, E.W. and Allen, D.G. (2004) Stretch-Activated Channels in Stretch-Induced Muscle Damage: Role in 
Muscular Dystrophy. Clinical and Experimental Pharmacology \& Physiology, 31, 551-556.

https://doi.org/10.1111/j.1440-1681.2004.04027.x

13. Yeung, E.W., Whitehead, N.P., Suchyna, T.M., Gottlieb, P.A., Sachs, F. and Allen, D.G. (2005) Effects of Stretch-Activated Channel Blockers on [Ca2+]i and Muscle Damage in the Mdx Mouse. The Journal of Physiology, 562, 367-380. https://doi.org/10.1113/jphysiol.2004.075275

14. Zhang, B.T., Yeung, S.S., Allen, D.G., Qin, L. and Yeung, E.W. (2008) Role of the Calcium-Calpain Pathway in Cytoskeletal Damage after Eccentric Contractions. Journal of Applied Physiology, 105, 352-357. https://doi.org/10.1152/japplphysiol.90320.2008

15. Chen, T.C., Chen, H.L., Lin, M.J., Wu, C.J. and Nosaka, K. (2009) Muscle Damage Responses of the Elbow Flexors to Four Maximal Eccentric Exercise Bouts Performed Every 4 Weeks. European Journal of Applied Physiology, 106, 267-275. https://doi.org/10.1007/s00421-009-1016-7

16. Ebbeling, C.B. and Clarkson, P.M. (1990) Muscle Adaptation Prior to Recovery Following Eccentric Exercise. European Journal of Applied Physiology and Occupational Physiology, 60, 26-31. https://doi.org/10.1007/BF00572181

17. Lavender, A.P. and Nosaka, K. (2008) A Light Load Eccentric Exercise Confers Protection against a Subsequent Bout of More Demanding Eccentric Exercise. Journal of Science and Medicine in Sport, 11, 291-298. https://doi.org/10.1016/j.jsams.2007.03.005

18. Nosaka, K. and Clarkson, P.M. (1995) Muscle Damage Following Repeated Bouts of High Force Eccentric Exercise. Medicine and Science in Sports and Exercise, 27, 1263-1269. https://doi.org/10.1249/00005768-199509000-00005

19. Nosaka, K., Sakamoto, K., Newton, M. and Sacco, P. (2001) How Long Does the Protective Effect on Eccentric Exercise-Induced Muscle Damage Last? Medicine and Science in Sports and Exercise, 33, 1490-1495. https://doi.org/10.1097/00005768-200109000-00011

20. Lehti, T.M., Kalliokoski, R. and Komulainen, J. (2007) Repeated Bout Effect on the Cytoskeletal Proteins Titin, Desmin, and Dystrophin in Rat Skeletal Muscle. Journal of Muscle Research and Cell Motility, 28, 39-47. https://doi.org/10.1007/s10974-007-9102-0

21. Takagi, R., Ogasawara, R., Tsutaki, A., Nakazato, K. and Ishii, N. (2016) Regional Adaptation of Collagen in Skeletal Muscle to Repeated Bouts of Strenuous Eccentric Exercise. Pflugers Archiv. European Journal of Physiology, 468, 1565-1572. https://doi.org/10.1007/s00424-016-1860-3

22. Takagi, R., Ogasawara, R., Takegaki, J., Tamura, Y., Tsutaki, A., Nakazato, K. and Ishii, N. (2018) Past Injurious Exercise Attenuates Activation of Primary Calcium-Dependent Injury Pathways in Skeletal Muscle during Subsequent Exercise. Physiological Reports, 6, e13660. https://doi.org/10.14814/phy2.13660

23. Corona, B.T., Balog, E.M., Doyle, J.A., Rupp, J.C., Luke, R.C. and Ingalls, C.P. (2010) Junctophilin Damage Contributes to Early Strength Deficits and EC Coupling Failure after Eccentric Contractions. American Journal of Physiology Cell Physiology, 298, C365-376. https://doi.org/10.1152/ajpcell.00365.2009

24. Mori, T., Agata, N., Itoh, Y., Miyazu-Inoue, M., Sokabe, M., Taguchi, T. and Kawakami, K. (2014) Stretch Speed-Dependent Myofiber Damage and Functional Deficits in Rat Skeletal Muscle Induced by Lengthening Contraction. Physiological Reports, 2, e12213. https://doi.org/10.14814/phy2.12213

25. Gissel, H. and Clausen, T. (1999) Excitation-Induced Ca2+ Uptake in Rat Skeletal Muscle. The American Journal of Physiology, 276, R331-339. https://doi.org/10.1152/ajpregu.1999.276.2.R331

26. Lieber, R.L. and Fridén, J. (1988) Selective Damage of Fast Glycolytic Muscle Fibres with Eccentric Contraction of the Rabbit Tibialis Anterior. Acta Physiologica Scandinavica, 133, 587-588. https://doi.org/10.1111/j.1748-1716.1988.tb08446.x 
27. Mori, T., Agata, N., Itoh, Y., Inoue-Miyazu, M., Mizumura, K., Sokabe, M., Taguchi, T. and Kawakami, K. (2018) Post-Injury Stretch Promotes Recovery in a Rat Model of Muscle Damage Induced by Lengthening Contractions. The Journal of Physiological Sciences, 68, 483-492. https://doi.org/10.1007/s12576-017-0553-9

28. Smith, H.K., Plyley, M.J., Rodgers, C.D. and McKee, N.H. (1999) Expression of Developmental Myosin and Morphological Characteristics in Adult Rat Skeletal Muscle Following Exercise-Induced Injury. European Journal of Applied Physiology and Occupational Physiology, 80, 84-91. https://doi.org/10.1007/s004210050562

29. Carraro, U., Boncompagni, S., Gobbo, V., Rossini, K., Zampieri, S., Mosole, S., Ravara, B., Nori, A., Stramare, R., Ambrosio, F., Piccione, F., Masiero, S., Vindigni, V., Gargiulo, P., Protasi, F., Kern, H., Pond, A. and Marcante, A. (2015) Persistent Muscle Fiber Regeneration in Long Term Denervation. Past, Present, Future. European Journal of Translational Myology, 25, 4832. https://doi.org/10.4081/bam.2015.2.77

30. Kern, H., Boncompagni, S., Rossini, K., Mayr, W., Fano, G., Zanin, M.E., Podhorska-Okolow, M., Protasi, F. and Carraro, U. (2004) Long-Term Denervation in Humans Causes Degeneration of Both Contractile and Excitation-Contraction Coupling Apparatus, Which Is Reversible by Functional Electrical Stimulation (FES): A Role for Myofiber Regeneration? Journal of Neuropathology and Experimental Neurology, 63, 919-931. https://doi.org/10.1093/jnen/63.9.919

31. Lovering, R.M. and De Deyne, P.G. (2004) Contractile Function, Sarcolemma Integrity, and the Loss of Dystrophin after Skeletal Muscle Eccentric Contraction-Induced Injury. American Journal of Physiology Cell Physiology, 286, C230-238. https://doi.org/10.1152/ajpcell.00199.2003

32. Tamaki, H., Yotani, K., Ogita, F., Hayao, K., Kirimto, H., Onishi, H., Kasuga, N. and Yamamoto, N. (2019) Low-Frequency Electrical Stimulation of Denervated Skeletal Muscle Retards Muscle and Trabecular Bone Loss in Aged Rats. International Journal of Medical Sciences, 16, 822-830. http://www.medsci.org/v16p0822.htm https://doi.org/10.7150/ijms.32590

33. Tamaki, H., Yotani, K., Ogita, F., Sugawara, K., Kirimto, H., Onishi, H., Kasuga, N. and Yamamoto, N. (2015) Effect of Electrical Stimulation-Induced Muscle Force and Streptomycin Treatment on Muscle and Trabecular Bone Mass in Early-Stage Disuse Musculoskeletal Atrophy. Journal of Musculoskeletal \& Neuronal Interactions, 15, 270-278.

34. Lynch, G.S., Fary, C.J. and Williams, D.A. (1997) Quantitative Measurement of Resting Skeletal Muscle [Ca2+]i Following Acute and Long-Term Downhill Running Exercise in Mice. Cell Calcium, 22, 373-383. https://doi.org/10.1016/S0143-4160(97)90022-1

35. Besarab, A., DeGuzman, A. and Swanson, J.W. (1981) Effect of Albumin and Free Calcium Concentrations on Calcium Binding in Vitro. Journal of Clinical Pathology, 34, 1361-1367. https://doi.org/10.1136/jcp.34.12.1361

36. Verburg, E., Murphy, R.M., Richard, I. and Lamb, G.D. (2009) Involvement of Calpains in Ca2+-Induced Disruption of Excitation-Contraction Coupling in Mammalian Skeletal Muscle Fibers. American Journal of Physiology Cell Physiology, 296, C1115-1122. https://doi.org/10.1152/ajpcell.00008.2009

37. Zhang, B.T., Whitehead, N.P., Gervasio, O.L., Reardon, T.F., Vale, M., Fatkin, D., Dietrich, A., Yeung, E.W. and Allen, D.G. (2012) Pathways of $\mathrm{Ca}(2)(+)$ Entry and Cytoskeletal Damage Following Eccentric Contractions in Mouse Skeletal Muscle. Journal of Applied Physiology, 112, 2077-2086. https://doi.org/10.1152/japplphysiol.00770.2011

38. Chapman, D., Newton, M., Sacco, P. and Nosaka, K. (2006) Greater Muscle Damage Induced by Fast versus Slow Velocity Eccentric Exercise. International Journal of Sports Medicine, 27, 591-598. https://doi.org/10.1055/s-2005-865920

39. Song, H., Nakazato, K. and Nakajima, H. (2004) Effect of Increased Excursion of the Ankle on the Severity of Acute Eccentric Contraction-Induced Strain Injury in the Gastrocnemius: An in Vivo Rat Study. The American Journal of Sports Medicine, 32, 1263-1269. https://doi.org/10.1177/0363546503262199 
40. Benfenati, V., Caprini, M., Dovizio, M., Mylonakou, M.N., Ferroni, S., Ottersen, O.P. and Amiry-Moghaddam, M. (2011) An Aquaporin-4/Transient Receptor Potential Vanilloid 4 (AQP4/TRPV4) Complex Is Essential for Cell-Volume Control in Astrocytes. Proceedings of the National Academy of Sciences of the United States of America, 108, 2563-2568. https://doi.org/10.1073/pnas.1012867108

41. Jo, A.O., Ryskamp, D.A., Phuong, T.T., Verkman, A.S., Yarishkin, O., MacAulay, N. and Krizaj, D. (2015) TRPV4 and AQP4 Channels Synergistically Regulate Cell Volume and Calcium Homeostasis in Retinal Muller Glia. The Journal of Neuroscience, 35, 13525-13537. https://doi.org/10.1523/JNEUROSCI.1987-15.2015

42. Crosbie, R.H., Dovico, S.A., Flanagan, J.D., Chamberlain, J.S., Ownby, C.L. and Campbell, K.P. (2002) Characterization of Aquaporin-4 in Muscle and Muscular Dystrophy. FASEB Journal: Official Publication of the Federation of American Societies for Experimental Biology, 16, 943-949. https://doi.org/10.1096/fj.01-0327com

43. Jimi, T., Wakayama, Y., Murahashi, M., Shibuya, S., Inoue, M., Hara, H., Matsuzaki, Y. and Uemura, N. (2000) Aquaporin 4: Lack of mRNA Expression in the Rat Regenerating Muscle Fiber under Denervation. Neuroscience Letters, 291, 93-96. https://doi.org/10.1016/S0304-3940(00)01382-3

44. Takekura, H., Tamaki, H., Nishizawa, T. and Kasuga, N. (2003) Plasticity of the Transverse Tubules Following Denervation and Subsequent Reinnervation in Rat Slow and Fast Muscle Fibres. Journal of Muscle Research and Cell Motility, 24, 439-451. https://doi.org/10.1023/A:1027356912404

45. Lee, K., Kouzaki, K., Ochi, E., Kobayashi, K., Tsutaki, A., Hiranuma, K., Kami, K. and Nakazato, K. (2014) Eccentric Contractions of Gastrocnemius Muscle-Induced Nerve Damage in Rats. Muscle \& Nerve, 50, 87-94. https://doi.org/10.1002/mus.24120

46. Hyldahl, R.D., Chen, T.C. and Nosaka, K. (2017) Mechanisms and Mediators of the Skeletal Muscle Repeated Bout Effect. Exercise and Sport Sciences Reviews, 45, 24-33. https://doi.org/10.1249/JES.0000000000000095

47. McHugh, M.P. (2003) Recent Advances in the Understanding of the Repeated Bout Effect: The Protective Effect against Muscle Damage from a Single Bout of Eccentric Exercise. Scandinavian Journal of Medicine \& Science in Sports, 13, 88-97. https://doi.org/10.1034/j.1600-0838.2003.02477.x 